\title{
The importance of fetuin-A in vascular calcification in children with chronic kidney disease
}

\author{
Irena Makulska, ${ }^{1, A-D}$, Maria Szczepańska 2,B,D, Dorota Drożdż3,B, \\ Dorota Polak-Jonkisz ${ }^{1, B}$, Danuta Zwolińska ${ }^{1, A, C-F}$ \\ ${ }^{1}$ Department of Pediatric Nephrology, Faculty of Medicine, Wroclaw Medical University, Poland \\ ${ }^{2}$ Department of Pediatrics in Zabrze, Medical University of Silesia, Katowice, Poland \\ ${ }^{3}$ Dialysis Unit, Jagiellonian University Medical College, Kraków, Poland \\ A - research concept and design; B - collection and/or assembly of data; $C$ - data analysis and interpretation; \\ $D$ - writing the article; $E$ - critical revision of the article; $F$ - final approval of the article
}

\section{Address for correspondence \\ Irena Makulska \\ E-mail: im.makulscy@gmail.com}

Funding sources

None declared

\section{Conflict of interest}

None declared

Received on September 23, 2017

Reviewed on January 1, 2018

Accepted on January 10, 2018

Published online on August 2, 2018

Cite as

Makulska I, Szczepańska M, Drożdż D, Polak-Jonkisz D,

Zwolińska D. The importance of fetuin-A in vascular calcification in children with chronic kidney disease. Adv Clin Exp Med. 2019;28(4):499-505. doi:10.17219/acem/82517

DOI

10.17219/acem/82517

\section{Copyright}

Copyright by Author(s)

This is an article distributed under the terms of the

Creative Commons Attribution Non-Commercial License

(http://creativecommons.org/licenses/by-nc-nd/4.0/)

\section{Abstract}

Background. The status of the cardiovascular (CV) system in children with chronic kidney disease (CKD) is significantly influenced by increasing stiffness of the arterial wall. This largely depends on the shortage of local and systemic inhibitors of soft tissue calcification.

Objectives. The aim of the study was to evaluate the role of fetuin-A in conjunction with other factors in the progressive hardening of the vascular wall in these children. We examined serum fetuin-A concentrations in relation to renal function, dialysis modality, and other clinical and biochemical markers promoting vascular calcification.

Material and methods. Twenty children on peritoneal dialysis (PD), 20 on hemodialysis (HD), 36 treated conservatively, and 26 healthy subjects were enrolled into a cross-sectional study. In all children, fetuin-A and numerous clinical and biochemical parameters were measured.

Results. The fetuin-A concentration was significantly lower in children on hemodialysis (HD) vs children on peritoneal dialysis (PD), conservatively treated subjects, and the control group. In sick children, fetuin-A concentration negatively correlated with dialysis vintage, PWV/ht, phosphate concentration, calcium phosphate product (CaxP), cumulative doses of calcium, and vitamin $\mathrm{D}_{3}$. In the whole study population, fetuin-A negatively correlated with blood pressure (BP), pulse wave velocity indexed to height (PWV/ht), intact parathyroid hormone (iPTH), high sensitivity (-reactive protein (hsCRP), and cholesterol concentrations.

Conclusions. In children with CKD, the decreased concentration of fetuin-A is related to other vascular calcification risk factors. Serum fetuin-A concentration may play a role in the identification of vascular disease risk factors in this population.

Key words: children, chronic kidney disease, fetuin-A 


\section{Introduction}

The status of the cardiovascular (CV) system in children with chronic kidney disease (CKD) depends largely on the growth in vessel wall stiffness (atherosclerosis). Mönckeberg-type medial calcification leads to an exacerbation of arterial stiffness, resulting in a rise in systolic blood pressure (SBP) and a reduction in diastolic blood pressure (DBP), as well as in an increase in pulse pressure value (PPV), which has been recognized as an independent risk factor for death from CV causes. ${ }^{1}$ In the course of progressive stiffening of the vessel wall, left ventricular hypertrophy, impairment of coronary blood flow and calcification of the heart valves are observed. All of these disorders lead to increased risk of myocardial infarction, arrhythmia and heart failure, and consequently, to sudden death. ${ }^{2,3}$ In patients with CKD, additional risk factors are disturbed calcium metabolism, phosphate $(\mathrm{P})$, high parathyroid hormone (PTH) level, elevated calcium phosphate product (CaxP), the number of episodes of hypercalcemia, and doses of calcium phosphate binders. ${ }^{4,5}$ The promoting factors of the formation of calcifications are long-lasting hypercalcemia and hyperphosphatemia, but the severity of changes largely depends on genetic predisposition. Block et al. reported on the negative correlation between the degree of calcification of the vascular wall and bone turnover. ${ }^{6}$ Similarly, other authors have shown that patients on dialysis who have had low levels of intact PTH (iPTH) and low alkaline phosphatase activity, the degree of calcification increases faster than in patients with high levels of iPTH. ${ }^{4}$ In light of recent reports, it is considered that the calcification of blood vessels is an active process that is initiated by hyperphosphatemia and is associated with deficient endogenous inhibitors of calcification. ${ }^{7,8}$ The basic structure responsible for this process is vascular smooth muscle cells (VSMC). Chronic kidney disease patients, especially patients on chronic dialysis, are deficient in systemic and local soft tissue calcification inhibitors.

The greatest interest of researchers is currently focused on fetuin-A ( $\alpha 2$-Heremans-Schmid-glycoprotein), the main systemic inhibitor of calcification in soft tissues. Synthesized in the liver, it reaches a high concentration in extracellular fluid, including plasma (above $1 \mathrm{~g} / \mathrm{L}$ ). Fetuin-A shows a negative relationship with inflammatory markers. The results of experimental studies demonstrate its important role in the inhibition of vascular wall calcification. It has been shown that mice lacking the gene for fetuin-A have severe metastatic calcifications of the soft tissues. ${ }^{9}$ The action of fetuin-A is performed through the binding of small calcium phosphate crystals, thus inhibiting their growth and deposition in tissues. It was found that in the presence of fetuin-A, a soluble complex called calcyprotein is formed, comprised of 8 molecules of fetuin-A ( $80 \%$ of the complex by weight), 1 molecule of matrix Gla protein (MGP) (2\% by weight), 790 atoms of calcium, and 580 molecules of phosphate (18\% by weight). ${ }^{10}$ The fetuin-A is able to inhibit the activity of TGF- $\beta$ and macrophages, and thereby to reduce the release of proinflammatory cytokines. ${ }^{11}$ In the presence of calcium, it has an affinity for proteins located on the cell surface - annexin II and VI. Fetuin-A binds to them and enters the endosomes of smooth muscle cells, thus inhibiting their apoptosis. The fetuin-A entering into the matrix vesicles and apoptotic bodies prevents soft tissue mineralization. It has also been found that fetuin-A accelerates the phagocytosis of apoptotic bodies and inhibits VSMC function and bone morphogenetic protein 2 function. ${ }^{12,13}$ Many investigators have shown that in patients with CKD, especially stage 5 , fetuin-A is significantly lower than in healthy individuals, which can affect the increased mortality from CV disease in these patients. ${ }^{14-17}$ Goodman et al. have studied this aspect of young adults (20-30 years of age) undergoing chronic hemodialysis (HD). Almost all patients (88\%) presented calcifications in the coronary arteries, especially those who started dialysis early in childhood. ${ }^{4}$ There have been only single reports of vascular calcification in children and adolescents with CKD. ${ }^{14,18-21}$

The aim of the study was to assess serum fetuin-A concentrations in children with CKD in relation to renal function, dialysis modality, and other clinical and biochemical markers promoting vascular calcification.

\section{Material and methods}

Seventy-six children with CKD, still asymptomatic of CV complications, were enrolled into the study. The patients were divided into 3 groups according to CKD stage and dialysis modality. Twenty-six age-matched children with primary nocturnal enuresis and normal kidney function served as controls. Clinical and demographic details of the study subjects are summarized in Table 1 . The $1^{\text {st }}$ group included 20 children on peritoneal dialysis (PD). Nocturnal intermittent peritoneal dialysis (NIPD) was performed in 12 patients and continuous cyclic peritoneal dialysis (CCPD) was administered in 8 patients. A Home-Choice machine was used (Baxter International, Inc., Deerfield, USA). Standard dialysis solutions with glucose concentrations of $1.5 \%$ and $2.3 \%$ and calcium concentrations of $1.25 \mathrm{mmol} / \mathrm{L}$ or $1.75 \mathrm{mmol} / \mathrm{L}$ were applied according to individual recommendations. The causes of CKD in children from the $1^{\text {st }}$ group (PD group) included structural urinary tract abnormalities $(n=9)$, glomerulonephritis $(n=5)$, polycystic kidney disease $(n=3)$, hereditary nephropathy $(n=2)$, and hemolytic uremic syndrome $(n=1)$. Fifteen children remained on hypotensive medication, including angiotensin-converting enzyme inhibitors (ACEI) $(n=13)$, calcium channel blockers $(n=6)$ and $\beta$-blockers $(n=3)$. All of the subjects received calcium-containing phosphate binders and vitamin D metabolites in doses adjusted to their requirements.

The $2^{\text {nd }}$ group (HD group) consisted of 20 children on maintenance HD. Dialysis sessions were performed 
Table 1. Clinical characteristics of the chronic kidney disease (CKD) groups and the controls (two-by-two comparison, p-values are shown)

\begin{tabular}{|c|c|c|c|c|c|}
\hline $\begin{array}{c}\text { Group } \\
\text { parameter }\end{array}$ & $\begin{array}{c}\text { PD } \\
n=20\end{array}$ & $\begin{array}{c}\text { HD } \\
n=20\end{array}$ & $\begin{array}{c}\text { Pre } \\
n=36\end{array}$ & $\begin{array}{c}\text { Controls } \\
n=26\end{array}$ & $p$-value \\
\hline Age [year] & $14.3 \pm 2.3$ & $15 \pm 3.3$ & $14.9 \pm 3.5$ & $14.5 \pm 3.3$ & NS \\
\hline Gender [male/female] & $12 / 8$ & $10 / 10$ & $17 / 19$ & $12 / 14$ & NS \\
\hline Dialysis vintage [months] & $12 \pm 11$ & $19 \pm 16$ & - & - & $p=0.02$ \\
\hline $\mathrm{BMI}\left[\mathrm{kg} / \mathrm{m}^{2}\right]$ & $18.7 \pm 3.9$ & $18.7 \pm 3.4$ & $20.6 \pm 4.1$ & $18.8 \pm 3.9$ & NS \\
\hline $\mathrm{SBP}[\mathrm{mm} \mathrm{Hg}]$ & $117 \pm 11^{1,3}$ & $128 \pm 13^{1,2,4}$ & $115 \pm 10^{1}$ & $100 \pm 9$ & $p<0.0001$ \\
\hline $\mathrm{DBP}[\mathrm{mm} \mathrm{Hg}]$ & $75 \pm 11^{1,3}$ & $82 \pm 10^{1,2,4}$ & $71 \pm 8^{1}$ & $64 \pm 6$ & $p<0.0001$ \\
\hline $\mathrm{PP}[\mathrm{mm} \mathrm{Hg}]$ & $42 \pm 9^{1}$ & $46 \pm 9^{1}$ & $44 \pm 8^{1}$ & $35 \pm 7$ & $p<0.0001$ \\
\hline $\mathrm{MBP}[\mathrm{mm} \mathrm{Hg}]$ & $86 \pm 12^{1,3}$ & $97 \pm 10^{1,2,4}$ & $86 \pm 7^{1,3}$ & $76 \pm 6$ & $p<0.0001$ \\
\hline Cumulative dose of calcium [g/kg] & $29.7 \pm 45.9$ & $46.6 \pm 45.8^{4}$ & $9.67 \pm 12.5^{3}$ & - & $p=0.0002$ \\
\hline Cumulative dose of vitamin $D_{3}[\mu \mathrm{g} / \mathrm{kg}]$ & $3.71 \pm 3.7$ & $4.76 \pm 3.37^{4}$ & $2.02 \pm 4.41^{3}$ & - & $p=0.0005$ \\
\hline PWV/ht $[\mathrm{m} / \mathrm{s}]$ & $5.51 \pm 0.68^{1,3}$ & $6.07 \pm 0.86^{1,2,4}$ & $5.53 \pm 0.69^{1,3}$ & $4.85 \pm 0.62$ & $p<0.0001$ \\
\hline
\end{tabular}

PD - peritoneal dialysis; HD - hemodialysis; Pre - predialysis; BMI - body mass index; SBP - systolic blood pressure; DBP - diastolic blood pressure; PP - pulse pressure; MBP - mean blood pressure; PWV/ht - pulse wave velocity indexed to height; NS - not significant.

${ }^{1}$ vs control group; ${ }^{2}$ vs PD group; ${ }^{3}$ vs HD group; ${ }^{4}$ vs Pre group.

3 times a week (3-5 h) using polysulfone membranes. The blood flow ranged from 120 to $250 \mathrm{~mL} / \mathrm{min}$, and dialysate flow did not exceed $500 \mathrm{~mL} / \mathrm{min}$. The dialysis fluid was buffered with bicarbonate and the calcium content was $1.25 \mathrm{mmol} / \mathrm{L}$ or $1.5 \mathrm{mmol} / \mathrm{L}$. The causes of CKD in this group were urinary tract abnormalities (CAKUT) $(n=12)$, glomerulonephritis $(n=6)$ and hereditary glomerulopathy $(\mathrm{n}=2)$. Nineteen hypertensive children were treated with ACEi $(\mathrm{n}=19)$, calcium channel blockers $(\mathrm{n}=11)$ and $\beta$-blockers $(n=2)$. All of the patients received calciumcontaining phosphate binders and vitamin D compounds.

The $3^{\text {rd }}$ group (Pre group) included 36 children with CKD (stages 2-4) on conservative treatment. The causes of CKD were urinary tract malformations $(n=22)$, glomerulonephritis $(n=5)$, polycystic kidney disease $(n=3)$, hereditary glomerulopathy $(n=2)$, unknown cause $(n=2)$, hemolytic uremic syndrome $(n=1)$, and complications after chemotherapy for cancer $(n=1)$. Stage 2 CKD was detected in 13 children, stage 3 in 10 and stage 4 in 13 . Twelve subjects were treated with ACE-I, 6 patients with angiotensin receptor blockers (ARB), 4 patients with calcium channel blockers, and 1 child received $\beta$-blockers.

All patients with stage 3 or 4 CKD as well as 5 children with stage 2 CKD received treatment with calcium-containing phosphate binders and vitamin D compounds.

Children under the age of 6 years and patients with diabetes or infection were excluded from the study. Informed consent for participation in the study was obtained from all of the parents and from children 15 years old or older. The research project was approved by the Wroclaw Medical University (Poland) ethics committee.

Selected biochemical and functional parameters in the study population were measured in order to assess the degree of vascular stiffness. In all patients and in the control group, the following biochemical parameters were determined: serum creatinine, albumin, acute phase proteins (high sensitivity C-reactive protein (hsCRP)), hemoglobin
(Hb), lipid count (total cholesterol (TCL), low-density lipoproteins cholesterol (LDL-cholesterol), high-density lipoproteins cholesterol (HGL-cholesterol), and triglycerides (TGL)), as well as parameters related to calcium $P$ metabolism (calcium, P and iPTH). The serum concentration of fetuin-A was also measured as a principal systemic inhibitor of soft tissue calcification.

As part of the assessment of vascular function, 24-h blood pressure (BP) was monitored with the evaluation of SBP, $\mathrm{DBP}$, mean blood pressure (MBP), pulse pressure (PP), and pulse wave velocity (PWV).

The stage of CKD was diagnosed based on the The National Kidney Foundation Kidney Disease Outcomes Quality (NKFK/DOQI) recommendations and the calculation of glomerular filtration rate (GFR) was performed using the Schwartz formula. ${ }^{22}$

In dialysis patients, the type and duration of renal replacement therapy was taken into account. In each patient, based on the measurement of height and weight, body mass index (BMI) was determined according to this formula: $\mathrm{BMI}=$ body weight $[\mathrm{kg}] /$ height $\left[\mathrm{m}^{2}\right]$.

Blood pressure was measured using a sphygmomanometer or an electronic DIANA device (DINAMAP, Boston, USA) for 24-hour monitoring. Measurements were taken in a seated position with the appropriate cuff selected to cover the width of $1 / 3$ of the length of the arm. Normal $\mathrm{BP}$ values were defined as values below the $90^{\text {th }}$ percentile and below the upper BP limit (according to the nomenclature defined in the Fourth Report on the Diagnosis, Evaluation, and Treatment of High Blood Pressure in Children and Adolescents) and prehypertension was defined as values between the $90^{\text {th }}$ and the $95^{\text {th }}$ percentile. ${ }^{23}$ For the evaluation of the results, percentile charts relating to gender, age and growth percentile from the National High Blood Pressure Education Program Working Group on High Blood Pressure in Children and Adolescents, Third Report, were used. ${ }^{24}$ The percentile growth charts we used 
in our study were developed by the Institute of Mother and Child in Warszawa, Poland. Mean blood pressure and PP were calculated according to these formulas: $\mathrm{MBP}=\mathrm{DBP}$ $+[(\mathrm{SBP}-\mathrm{DBP}) / 3]$ and $\mathrm{PP}=\mathrm{SBP}-\mathrm{DBP}$. In each patient, the cumulative dose of oral calcium in the gastrointestinal tract, mostly taken in the form of calcium-containing phosphate binders, was calculated. The cumulative dose of the active form of vitamin $\mathrm{D}_{3}$ which each patient had previously received was also calculated.

Blood samples were collected in the morning, under fasting conditions, while performing other routine tests. In order to determine the concentration of creatinine, albumin, $\mathrm{Hb}$, acute phase protein (hsCRP), TCL, LDL-cholesterol, HDL-cholesterol, TGL, calcium, and phosphate, blood was simultaneously drawn and the serum parameters were assayed on the same day. For fetuin-A, blood was drawn into dry tubes, then centrifuged at 3,000 rpm for $15 \mathrm{~min}$ and the serum was frozen at $-20^{\circ} \mathrm{C}$ until assayed. The blood used in $\mathrm{iPTH}$ testing was collected into tubes containing ethylenediaminetetraacetic acid (EDTA) and centrifuged for $15 \mathrm{~min}$ at 1,000 rpm, within $30 \mathrm{~min}$ of sampling. After the separation of plasma from cellular components, the plasma was frozen and stored at $-20^{\circ} \mathrm{C}$ until assayed. Serum creatinine, albumin, calcium, phosphate, lipid, and hsCRP levels, as well as a complete blood count, were assessed in Central Laboratory, University Teaching Hospital in Wrocław, Poland, with standard methods. The iPTH concentration was determined by IRMA using Duo PTH manual kits (Scantibodies Laboratory, Inc., Santee, USA) (standard laboratory normal value: $14-66 \mathrm{pg} / \mathrm{mL}$ ). The measurement of fetuin-A was performed twice and the mean values were analyzed. Fetuin-A was determined by enzyme-linked immunosorbent assay (ELISA) using a commercially available kit (BioVendor, Brno-̌̌ečkovice a Mokrá Hora, Czech Republic). The PWV study was performed according to the methodology we presented in an earlier work. ${ }^{25}$

Statistical analyses were performed using the STATGRAPHICS package (Centurion XV v. 15.2.06, StatPoint, Inc. Herndon, USA). The results were expressed as mean values \pm standard deviation (SD) when a normal distribution of variables was obtained. The differences were then compared by an analysis of variance (ANOVA) test. In the case of non-normal distribution, a nonparametric Kruskal-Wallis test for median values was used. For the evaluation of the relationship between parameters with a normal distribution of variables, Pearson's correlation test was performed. Spearman's test was applied for data with non-normal distribution. Since the p-value in the
ANOVA table was less than 0.05, there was a statistically significant relationship between the variables. A p-value of less than 0.05 in other tests was considered statistically significant.

\section{Results}

The study groups were not found to differ significantly with respect to age, sex or BMI. The concentration of fetuin-A was the lowest in the HD group and was significantly different from the concentrations of the PD group, the Pre group and the group of healthy subjects. In patients undergoing PD, fetuin-A concentration was significantly lower than in Pre group patients and in the healthy controls. The highest values of this calcification inhibitor were detected in the control group. Detailed data are shown in Table 2.

The highest BP, SBP, DBP, and MBP values were found in the group of children on HD. They varied significantly compared to the group of healthy children, to the PD group and to the Pre group. Pulse pressure was significantly higher in all groups of children with CKD in comparison to healthy peers, regardless of the stage of the disease or the methods of renal replacement therapy. The duration of PD was shorter than the time of chronic HD treatment. Children in the HD group received the highest cumulative dose (per kg of body weight) of both calcium carbonate and active metabolites of vitamin $\mathrm{D}_{3}$. The difference was statistically significant in comparison to the children in the Pre group. The highest values of PWV/ht were noted in HD patients, and they differed significantly from the results obtained in all other groups. Pulse wave velocity indexed to height (PWV/ht) measurements were also significantly higher in the PD group and in the Pre group than in the control group. Detailed data are presented in Table 1.

Significantly lower albumin concentration and higher total cholesterol and triglyceride levels were observed in PD and Pre groups than in healthy controls. The highest concentration of LDL-cholesterol and total cholesterol, found in the PD group, differed significantly from the values recorded in other groups. In all CKD groups, Hb levels were lower than in the healthy subjects. The most pronounced decrease in $\mathrm{Hb}$ concentration was observed in the HD group. The lowest concentration of calcium and the highest of phosphate were found in the HD group. These values were significantly different from the other groups.

Table 2. Fetuin-A concentration in the study groups (two-by-two comparison, p-values are shown)

\begin{tabular}{|c|c|c|c|c|c|}
\hline $\begin{array}{c}\text { Group } \\
\text { parameter }\end{array}$ & $\begin{array}{c}\text { PD } \\
n=20\end{array}$ & $\begin{array}{c}H D \\
n=20\end{array}$ & $\begin{array}{c}\text { Pre } \\
n=36\end{array}$ & $\begin{array}{l}\text { Control } \\
n=26\end{array}$ & p-value \\
\hline Fetuin-A [ng/mL] & $56.67 \pm 0.55^{1,3,4}$ & $40.73 \pm 0.96^{1,2,4}$ & $63.38 \pm 0.88^{1,2,3}$ & $98.56 \pm 0.95$ & $p<0.0001$ \\
\hline
\end{tabular}

PD - peritoneal dialysis; HD - hemodialysis; Pre - predialysis.

${ }^{1}$ vs control group; ${ }^{2}$ vs PD group; ${ }^{3}$ vs HD group; ${ }^{4}$ vs Pre group. 
Table 3. Biochemical characteristics of the chronic kidney disease (CKD) groups and the controls (two-by-two comparison, p-values are shown)

\begin{tabular}{|c|c|c|c|c|c|}
\hline $\begin{array}{c}\text { Group } \\
\text { parameter }\end{array}$ & $\begin{array}{c}\text { PD } \\
n=20\end{array}$ & $\begin{array}{c}H D \\
n=20\end{array}$ & $\begin{array}{c}\text { Pre } \\
n=36\end{array}$ & $\begin{array}{c}\text { Controls } \\
n=26\end{array}$ & $\mathrm{p}$-value \\
\hline $\mathrm{Hb}[\mathrm{g} / \mathrm{dL}]$ & $11.14 \pm 1.23^{1}$ & $10.19 \pm 1.34^{1,4}$ & $11.99 \pm 2.07^{1,3}$ & $13.3 \pm 1.19$ & $p<0.0001$ \\
\hline Albumin $[\mathrm{g} / \mathrm{dL}]$ & $3.9 \pm 0.34^{1}$ & $4.0 \pm 0.35$ & $3.9 \pm 0.68^{1}$ & $4.3 \pm 0.44$ & $p=0.009$ \\
\hline Total cholesterol [mg/dL] & $206 \pm 43^{1}$ & $174 \pm 33$ & $203 \pm 100^{1}$ & $162 \pm 13$ & $p=0.0001$ \\
\hline LDL-cholesterol [mg/dL] & $143 \pm 44^{1,3,4}$ & $92 \pm 31^{2}$ & $105 \pm 69^{2}$ & $84 \pm 6.2$ & $p<0.0001$ \\
\hline HDL-cholesterol [mg/dL] & $52 \pm 15$ & $46 \pm 9$ & $50 \pm 10$ & $49 \pm 4$ & NS \\
\hline TGL [mg/dL] & $179 \pm 75^{1}$ & $166 \pm 59$ & $187 \pm 242^{1}$ & $86 \pm 6.5$ & $p<0.0001$ \\
\hline Calcium [mg/dL] & $9.71 \pm 0.79^{3}$ & $9.23 \pm 0.56^{1,2,4}$ & $9.69 \pm 0.63^{1,3}$ & $9.99 \pm 0.34$ & $p=0.0002$ \\
\hline$P[\mathrm{mg} / \mathrm{dL}]$ & $5.48 \pm 1.81^{1,3,4}$ & $6.32 \pm 1.17^{1,2,4}$ & $4.52 \pm 0.97^{2,3}$ & $4.45 \pm 0.54$ & $p<0.0001$ \\
\hline $\mathrm{Ca} \times \mathrm{P}\left[\mathrm{mg}^{2} / \mathrm{dL}^{2}\right]$ & $53.5 \pm 19.4^{1,4}$ & $58.1 \pm 10^{1,4}$ & $43.6 \pm 8.9^{2,3}$ & $44.3 \pm 5.3$ & $p<0.0001$ \\
\hline ¡PTH [pg/mL] & $206 \pm 1771,3$ & $444 \pm 524^{1,2,4}$ & $138 \pm 135^{3}$ & $30.7 \pm 4$ & $p<0.0001$ \\
\hline $\mathrm{hsCRP}[\mathrm{mg} / \mathrm{L}]$ & $2.97 \pm 0.45^{3}$ & $3.64 \pm 0.98^{1,2}$ & $3.3 \pm 0.65$ & $2.5 \pm 0.15$ & $p<0.0001$ \\
\hline Creatinine [mg/dL] & $6.37 \pm 2.6^{1,4}$ & $7.6 \pm 3.4^{1,4}$ & $2.5 \pm 1.6^{1,2,3}$ & $0.68 \pm 0.11$ & $p<0.0001$ \\
\hline
\end{tabular}

PD - peritoneal dialysis; HD - hemodialysis; Pre - predialysis; TCL - total cholesterol; LDL-cholesterol - low-density lipoproteins cholesterol; HDL-cholesterol - high-density lipoproteins cholesterol; TGL - triglycerides; P - phosphate; CaxP - calcium phosphate product; iPTH - intact parathormone; hsCRP - high sensitivity C-reactive protein; NS - not significant.

${ }^{1}$ vs control group; ${ }^{2}$ vs PD group; ${ }^{3}$ vs HD group; ${ }^{4}$ vs Pre group.

Table 4. Analyses of factors correlated with fetuin-A in CKD patients (PD + $\mathrm{HD}+$ Pre) (Pearson correlation test)

\begin{tabular}{|l|c|c|}
\multicolumn{1}{|c|}{ Variable } & $r$ & $p$-value \\
\hline Fetuin-A dialysis vintage & $r=-0.34$ & $p=0.002$ \\
\hline$P$ & $r=-0.23$ & $p=0.03$ \\
\hline CaxP & $r=-0.27$ & $p=0.01$ \\
\hline Cumulative dose of calcium & $r=-0.22$ & $p=0.04$ \\
\hline Cumulative dose of vitamin $D_{3}$ & $r=-0.26$ & $p=0.02$ \\
\hline PWV/ht & $r=-0.47$ & $p<0.0001$ \\
\hline
\end{tabular}

P - phosphate; CaxP - calcium phosphate product; PWV/ht - pulse wave velocity indexed to height; $r$ - correlation coefficient.

The values of CaxP in children with CKD on dialysis treatment were significantly higher than in the control group and in patients treated conservatively (Pre group), but did not differ regarding dialysis modality. The highest values of iPTH levels were found in the HD group; they differed significantly in relation to other groups. The CRP concentration was also highest in the HD group and was significantly different from those observed in the control group and the PD group. There were no significant differences in CRP levels between the Pre group and the control group or the PD group. Detailed data is shown in Table 3. In the total population of children with CKD (PD + HD + Pre), we found statistically significant negative linear correlations of fetuin-A levels with PWV/ht, dialysis duration, $\mathrm{P}$, CaxP, and the cumulative doses of calcium and vitamin $\mathrm{D}_{3}$. Detailed data is presented in Table 4.

In the entire study population, we observed similar correlations regarding fetuin-A among all patients. In addition in the whole analyzed population, we found a negative linear correlation between fetuin-A and blood pressure values (SBP, DBP, PP, and MBP), iPTH, hsCRP, total
Table 5. Analyses of factors correlated with fetuin-A in the whole study population (Pearson correlation test)

\begin{tabular}{|l|r|c|}
\multicolumn{1}{|c|}{ Variable } & $r$ & $p$-value \\
\hline Fetuin-A SBP & $r=-0.53$ & $p<0.0001$ \\
\hline DBP & $r=-0.44$ & $p<0.0001$ \\
\hline PP & $r=-0.33$ & $p=0.0006$ \\
\hline MBP & $r=-0.44$ & $p<0.0001$ \\
\hline P & $r=-0.32$ & $p=0.0009$ \\
\hline Ca & $r=0.24$ & $p=0.01$ \\
\hline CaxP & $r=-0.28$ & $p=0.003$ \\
\hline iPTH & $r=-0.31$ & $p=0.001$ \\
\hline Cumulative dose of calcium & $r=-0.35$ & $p=0.0002$ \\
\hline Cumulative dose of vitamin $D_{3}$ & $r=-0.41$ & $p<0.0001$ \\
\hline Total cholesterol & $r=-0.21$ & $p=0.03$ \\
\hline LDL-cholesterol & $r=-0.22$ & $p=0.02$ \\
\hline TGL & $r=-0.23$ & $p=0.01$ \\
\hline hsCRP & $r=-0.32$ & $p=0.0009$ \\
\hline PWV/ht & $r=-0.39$ & $p<0.0001$ \\
\hline
\end{tabular}

SBP - systolic blood pressure; DBP - diastolic blood pressure; PP - pulse pressure; MBP - mean blood pressure; $P$ - phosphate; CaxP - calcium phosphate product; iPTH - intact parathormone; LDL - low-density lipoproteins cholesterol; TGL - triglycerides; hsCRP - high sensitivity C-reactive protein; PWV/ht - pulse wave velocity indexed to height.

cholesterol, LDL-cholesterol, and TGL. Detailed data is presented in Table 5. In the control group, there were no statistically significant correlations with fetuin-A. Assessing the impact of a single HD session on serum fetuin-A concentration, a significant increase in the concentration of fetuin-A after HD was found. Detailed data is presented in Table 6 . 
Table 6. The impact of a single hemodialysis (HD) session on serum fetuin-A concentration

\begin{tabular}{|l|c|c|c|}
\hline \multicolumn{1}{|c|}{ Serum } & Before HD & After HD & $p$-value \\
\hline $\begin{array}{l}\text { Fetuin-A } \\
{[\mathrm{ng} / \mathrm{mL}]}\end{array}$ & $40.73 \pm 0.96$ & $52.41 \pm 0.58$ & $\mathrm{p}<0.0001$ \\
\hline
\end{tabular}

\section{Discussion}

The complex pathogenesis of vascular injury in the course of CKD also involves factors that promote and inhibit calcification of soft tissues. While the influence of stimulating factors such as calcium, P, PTH, vitamin $\mathrm{D}_{3}$, and calcium phosphate-binding formulas on this process is well-known, less has been documented about the role of calcification inhibitors. Fetuin-A, which creates about $50 \%$ of the total plasma pool of calcification-inhibiting compounds, has been the subject of interest in patients with CKD over the past few years. In this study, the concentration of fetuin-A was significantly lower in all examined children with CKD compared to the control group, but the biggest decrease was observed in HD group. This is consistent with the vast number of reports on the subject in adults. ${ }^{15,16,26,27}$ The explanation for the reduced levels of fetuin-A may be the status of chronic inflammation, which in patients with end-stage renal failure is the most severe causative factor.

The influence of inflammation on the concentration of serum fetuin-A is supported by the research of Dervisoglu et al., who showed a negative correlation between fetuin-A and the concentration of proinflammatory cytokines. ${ }^{28}$ In a group of adults on HD, a relationship was also found between low levels of fetuin-A and higher mortality from CV causes. On the other hand, Shroff et al. presented different results in children: an increase in circulating levels of fetuin-A in children undergoing dialysis compared to healthy peers. ${ }^{29}$ Lower concentrations of fetuin-A were observed only in patients with heart valve and coronary artery calcifications. But even in these cases, concentrations of fetuin-A were higher than those observed in the control group. Nevertheless, it should be noted that the average time on dialysis was nearly half as long in their study as in our population, which is probably not without significance.

Additionally, a negative correlation between serum fetuin-A and dialysis duration has also been demonstrated by other authors. According to Shroff et al., elevated levels of fetuin-A in children undergoing dialysis could be regarded as an initial phase of a systemic response to proinflammatory and hypercalcemic agents. The authors suggest that a prolonged stimulation of such factors can reduce the compensation mechanisms of the body and can lead to a reduction in the concentration of natural inhibitors of calcification..$^{29}$ On the other hand, Ziółkowska et al. examined 28 children on dialysis (either on HD or PD) and
43 healthy children, and found no significant difference in the concentration of fetuin-A between the 2 groups. ${ }^{30}$

The results of research on the levels of fetuin-A in PD patients are also ambiguous: different authors found both higher levels and the same levels in comparison to adult patients on HD. ${ }^{31,32}$ In our study population, we demonstrated significantly lower concentrations of this calcification inhibitor in children on PD than in patients treated conservatively. However, compared to children on HD, the values were significantly higher. This can be explained not only by the shorter dialysis duration, but also by the less severe disorders of calcium-phosphate metabolism compared to children on HD. The negative correlation between the concentration of phosphate, $\mathrm{CaxP}, \mathrm{iPTH}$, and fetuin-A in the group of all patients with CKD seems to confirm this hypothesis. In children treated conservatively, fetuin-A level was the highest, although it was still lower than in the control group. These results do not support the observations made by Schaible et al., who did not show such differences. ${ }^{14}$ In one of the few studies on pediatric kidney transplantation, Van Summerenet al. ${ }^{32}$ found a significant decrease in the concentration of fetuin- $\mathrm{A}$, in contrast to the observations of Schaible et al. ${ }^{14}$ It should be emphasized that the reports on predialysis patients and PD are limited and include heterogeneous groups regarding the amount of procalcemic factors, chronic inflammation and comorbid conditions, which partly explains these differences. Also, one cannot rule out genetic factors which, as shown by studies of the German authors, may individually affect the synthesis of these inhibitors. ${ }^{33}$ Also, it is important that fetuin-A is not only an inhibitor of calcification, but that it also plays other roles within the body. It acts as a negative acute phase protein which inhibits the overproduction of proinflammatory cytokines and modulates the activity of the insulin receptor, affecting insulin resistance. ${ }^{34}$

Regardless of these discrepancies, there is much evidence that fetuin-A plays a protective role against vascular calcification and increasing vascular stiffness. This is confirmed by both studies in animal models and by clinical data in patients with CKD, showing a negative correlation between fetuin-A concentration and the presence of calcifications in the coronary arteries, carotid artery intima-media thickness, and pulse wave acceleration. ${ }^{35-40}$ Fetuin-A proved to also be an independent predictor of stiffness and vascular calcification in children after renal transplantation. ${ }^{33}$ In our study, we demonstrated a significant negative correlation between serum fetuin-A and the values of PWV/ht, BP, calcium, and lipid metabolism. Ziółkowska et al. studied the influence of many factors responsible for the progression of blood vessel calcification - including indices of calcium and P metabolism, degree of bone turnover, and lipids in children at different stages of CKD, with and without vascular changes - and revealed that only fetuin-A was the differentiating factor. ${ }^{30}$

Our study has several limitations. Firstly, the sample size, particularly subgroups, may be insufficient to show 
significant differences. Our patients were recruited from 3 of the pediatric nephrology centers which treat children from the whole southern region of Poland. Further studies with larger sample sizes are necessary, especially in light of new therapeutic methods which enable the lowering of vascular stiffness in CKD pediatric patients.

It can be concluded that the reduced serum fetuin-A concentration which is particularly pronounced in dialyzed children and its relationship with other progressive arterial stiffness risk factors indicate a significant role of this calcification inhibitor in the development of $\mathrm{CV}$ complications in children with CKD.

\section{References}

1. London GM, Marchais SJ, Guerin AP, Metivier F, Adda H. Arterial structure and function in end-stage renal disease. Nephrol Dial Transplant. 2002;17(10):1713-1724.

2. London GM, Marchais SJ, Guerin AP. Arterial stiffness and function in end-stage renal disease. Adv Kidney Dis. 2004;11(2):202-209.

3. Quinibi WY. Reducing the burden of cardiovascular calcification in patients with chronic kidney disease. J Am Soc Nephrol. 2005; 16(Suppl 2):S95-S102.

4. Goodman WG, Goldin J, Kuizon BD, et al. Coronary-artery calcification in young adults with end-stage renal disease who are undergoing dialysis. N Engl J Med. 2000;342(20):1478-1483.

5. Oh J, Wunsch R, Turzer M, et al. Advanced coronary and carotid arteriopathy in young adults with childhood-onset chronic renal failure. Circulation. 2002;106(1):100-110.

6. Block GA, Klassen PS, Lazarus JM, Ofsthun N, Lowrie EG, Chertow GM. Mineral metabolism, mortality and morbidity in maintenance hemodialysis. J Am Soc Nephrol. 2004;15(8):2208-2218.

7. Deriki U, El Nahas M. Vascular calcifications in uremia: Old concept and new insights. Semin Dial. 2006;19(1):60-68.

8. Floege J, Ketteler M. Vascular calcification in patients with end-stage renal disease. Nephrol Dial Transplant. 2004;19(Suppl 5):V59-66.

9. Schaeffer C, Heiss C, Schwarz A, et al. The serum protein a2-Heremans-Schmid glycoprotein/fetuin-A is a systemically acting inhibitor of ectopic calcification. J Clin Invest. 2003;112(3):357-366.

10. Price PA, Thomas GR, Bardini AW, Figueira WF, Caputo JM, Williamson MK. Discovery of a high molecular weight complex of calcium, phosphate, fetuin and matrix gamma-carboxyglutamic acid protein in the serum of etidronate-treated rats. J Biol Chem. 2002;277(6):3926-3934.

11. Heiss A, DuChesne A, Denecke B, et al. Structural basis of calcification inhibition by a2-HS glycoprotein/fetuin-A. Formation of colloidal calciprotein particles. J Biol Chem. 2003;278(15):13333-13341.

12. Chen NX, O'Neill KD, Chen X, et al. Fetuin-A uptake in bovine vascular smooth muscle cells is calcium dependent and mediated by annexins. Am J Renal Physiol. 2007;292(2):F599-606.

13. Reynolds JL, Skepper JN, McNair, et al. Multifactorial roles for serum protein fetuin-A in inhibition of human vascular smooth muscle cell calcification. J Am Soc Nephrol. 2005;16(10):2920-2930.

14. Schaible J, Wigger M, Staude H, et al. Serum fetuin-A and vitamin D in children with mild-to-severe chronic kidney disease: A cross-sectional study. Nephrol Dial Transplant. 2012;27(3):1107-1113.

15. Scialla JJ, Kao WH, Crainiceanu C, et al. Biomarkers of vascular calcification and mortality in patients with ESRD. Clin J Am Soc Nephrol. 2014;9(4):745-755.

16. Ketteler $M$, Bongartz $P$, Westenfeld $R$, et al. Association of low fetuin-A levels (AHSG) concentrations in serum in patients on dialysis with cardiovascular mortality: A cross sectional study. Lancet. 2003;361(9360): 827-833.

17. Stenvinkel P, Wang K, Qureshi AR, et al. Low fetuin-A levels are associated with cardiovascular death: Impact of variations in the gene encoding fetuin. Kidney Int. 2005;67:2383-2392.

18. Sheth RD, Perez MD, Goldstein SL. Cardiovascular calcifications in pediatric patients receiving maintenance dialysis. Pediatr Nephrol. 2003;18(8):810-813.
19. Civilibal M, Caliskan S, Adaletli I, et al. Coronary artery calcifications in children with end-stage renal disease. Pediatr Nephrol. 2006;21(10): 1426-1433.

20. Bakiler AR, Yavascan O, Harputluoglu N, Kara OD, Aksu N. Evaluation of aortic stiffness in children with chronic renal failure. Pediatr Nephrol. 2007;22(11):1911-1919.

21. Collura G, Capozza N, Francalanci P, et al. Arterial changes in children undergoing renal transplantation. Trans Proceed. 2008;40:1891-1894.

22. Schwartz GJ, Gauthier B. A simple estimate of glomerular filtration rate in adolescent boys. J Pediatr. 1985:106(3):522-526.

23. The Fourth Report on the Diagnosis, Evaluation, and Treatment of High Blood Pressure in Children and Adolescents; U.S. Department of Health and Human Services; National Institutes of Health, National Heart, Lung, and Blood Institute NIH Publication No. 05-5267. 2005.

24. Mavroudis C, Backer CL. National High Blood Pressure Education Program Working Group on High Blood Pressure in Children. Pediatr Cardiac Surg. 2008;251.

25. Makulska I, Szczepańska M, Drożdż D, Polak-Jonkisz D, Zwolińska D. Skin autofluorescence as a marker of cardiovascular risk in children with chronic kidney disease. Pediatr Nephrol. 2013;28(1):121-128.

26. Coen G, Manni M, Agnoli A, et al. Cardiac calcifications: Fetuin-A and other risk factors in hemodialysis patients. ASAIO J. 2006;52(2): 150-156.

27. Porazko T, Kuźniar J, Kusztal M, et al. Increased aortic wall stiffness associated with low circulating fetuin-A and high C-reactive protein in predialysis patients. Nephron Clin Pract. 2009;113(2):81-87.

28. Dervisoglu E, Kir HM, Kalender B, Caglayan CO, Eraldemir CO. Serum fetuin-A concentrations are inversely related to cytokines concentrations in patients with chronic renal failure. Cytokine. 2008;44(3): 323-327.

29. Shroff RC, Shah V, Hiorns MP, et al. The circulation calcification inhibitors, fetuin-A and osteoprotegerin but not Matrix Gla protein are associated with vascular stiffness and calcification in children on dialysis. Nephrol Dial Transplant. 2008;23(10):3263-3271.

30. Ziółkowska H, Brzeski M, Roszkowska-Blaim M. Determinants of the intima media thickness in children and adolescents with chronic kidney disease. Pediatr Nephrol. 2008;23(5):805-811.

31. Hermans MM, Brandenburg V, Ketteler M, et al. Study on the relationship of serum fetuin-A concentration with aortic stiffness in patients on dialysis. Nephrol Dial Transplant. 2006;21(5):1293-1299.

32. van Summeren MJ, Hameleers JM, Schurgers LJ, et al. Circulating calcification inhibitors and vascular properties in children after renal transplantation. Pediatr Nephrol. 2008;23(6):985-993.

33. Jahnen-Dechent W, Schinke T, Trindl A, et al. Cloning and targeted deletion of the mouse fetuin gene. J Biol Chem. 1997;272(50): 31496-31503.

34. Eraso LH, Ginwala N, Qasim AN, et al. Association of lower plasma fetuin-A levels with peripheral arterial disease in type- 2 diabetes. Diabetes Care. 2010;33(2):408-410.

35. Westenfeld R, Schäfer C, Krüger T, et al. Fetuin-A protects against atherosclerotic calcification in CKD. J Am Soc Nephrol. 2009;20(6): 1264-1274.

36. Kirkpantur A, Altun B, Hazirolan T, et al. Association among serum fetuin-A level, coronary artery calcification and bone mineral densitometry in maintenance of hemodialysis patients. ArtifOrgans. 2009; 33(10):844-854

37. Moe SM, Reslerova M, Ketteler M, et al. Role of calcification inhibitors in the pathogenesis of vascular calcification in chronic kidney disease (CKD). Kidney Int. 2005;67(6):2295-2304.

38. Caglar K, Yilmaz MI, Saglam M, et al. Serum fetuin-A concentration and endothelial dysfunction in chronic kidney disease. Nephron Clin Pract. 2008;108(3):233-420.

39. Toussaint ND, Lau KK, Strauss BJ, Polkinghorne KR, Kerr PG. Associations between vascular calcification, arterial stiffness and bone mineral density in chronic kidney disease. Nephrol Dial Transplant. 2008;23(2):586-593.

40. Ford ML, Tomlinson LA, Smith ER, Rajkumar C, Holt SG. Fetuin-A is an independent determinant of change of aortic stiffness over 1 year in non-diabetic patients with CKD stage 3 and 4. Nephrol Dial Transplant. 2010;25(6):1853-1858. 